\title{
Development of E-commerce Based on the Study of Perceived Credit Risk Formation and Control Mechanism
}

\author{
En-lin $\mathrm{Li}^{1,2}$ \\ 1.Post-doctoral Research Working Station of North-east Asia Outsourcing Research \\ Center, Harbin University of Commerce, \\ 2. Management School, Harbin Institute of Technology \\ 50023588@qq.com
}

\begin{abstract}
Nowadays, e-commerce is more and more important in our lives, and developing more and more rapidly. Perceived credit risk is blocked the development and regarded as a key factor influencing consumer decision-making in the electronic commerce shopping. In this paper, a model based on system dynamic is built to analyze formation and control mechanism of perceived credit risk, countermeasures and suggestions are provided. The study is helpful to provide evidence to reduce credit risk, and promote the healthy development of e-commerce, which would be useful to develop e-commerce industry.
\end{abstract}

Keywords: electronic commerce; formation; control; decision-making

\section{Introduction}

The human society has passed through a lengthy nomadic farming, industry and commerce, the change of society, is gradually moving towards the information society, as of January 2013, the number of Internet users in China reached 565 million. Electronic commerce is the core and foundation of the information society, directly affects the development of information in every field of society, politics, economy, culture and so on, in recent years, electronic commerce is developing vigorously, e-commerce has become an important social and economic development trends of business model. Compared with the traditional markets, it is more difficult to build trust between buyers and sellers because of impersonal characteristics of online transactions, information asymmetry and time and space in ecommerce transaction process. Therefore, perceived credit become an important reference for the decision-making of exchange behavior. It is helpful to promote the healthy development of e-commerce by analyzing and studying the formation and control mechanism of ecommerce perceived credit risk.

\section{Literature Review}

Yehoshua Liebermann and ShmuelStashevsky(1998) summarized that previous research suggests that perceived risk is an important ingredient in the consumer decision-making process. The purpose of the present study is to investigate what are the perceived barriers to Internet usage and e-marketing by both users and non-users. By understanding these potential obstacles, more efficient marketing strategies will become available that will drive Internet use and e-commerce[1]. Dan J. Kim, Donald L. Ferrin and H. RaghavRao(2008) developed a theoretical framework describing the trust-based decision-making process a consumer uses when making a purchase from a given site, and tested the proposed model using a Structural 
Equation Modeling technique on Internet consumer purchasing behavior data collected via a Web survey, then considered the implications of the model. The results of the study show that Internet consumers' trust and perceived risk have strong impacts on their purchasing decisions. Consumer disposition to trust, reputation, privacy concerns, security concerns, the information quality of the website, and the company's reputation, have strong effects on Internet consumers' trust in the Website. Interestingly, the presence of a third-party seal did not strongly influence consumers' trust [2]. Carolina Lopez-Nicolas, Francisco Jose MolinaCastillo(2008) designed tools to gain a deeper understanding of Customer Knowledge Management (CKM) inside the e-commerce context. The relationship between the CKM literature and the e-commerce literature is evaluated through several user characteristics such as risk preference, Internet preference and Internet knowledge and their impact on customers' online perceived risk and purchase intentions depending on the presence of certain CKM tools on the web site. The empirical study is based on a survey of 276 customers with previous online experience. By using multidimensional analysis, this study shows that the customers' perceived risk associated with different CKM tools plays an important role in explaining certain customer online behaviour[3]. Cheol Park, Jong-Kun Jun, (2003) attempted to examine differences in Internet usage, Internet innovativeness, perceived risks of Internet buying, and Internet buying behaviors between Korea and America, and to identify a model for factors influencing Internet buying behavior, explained by Internet usage, perceived risks, and innovativeness on a cross-cultural basis. Results showed that there were significant differences in Internet usage and the perceived risks of Internet shopping, but no significant differences in Internet buying intentions or online buying experience between Korean and American consumers. Nonetheless, analyzing a regression model of factors influencing Internet buying behavior, and cultural differences in effects of Internet usage and perceived risks on Internet buying behavior were found. While there were main effects of Internet usage and perceived risk on Internet buying behavior, these effects were weaker or even opposite to those related to Korean samples. The implications of the study are discussed and further research was suggested[4]. Anthony D. Miyazaki, Ana Fernandez(2001) found out that Risk perceptions regarding Internet privacy and security have been identified as issues for both new and experienced users of Internet technology. This paper explores risk perceptions among consumers of varying levels of Internet experience and how these perceptions relate to online shopping activity. Findings provide evidence of hypothesized relationships among consumers' levels of Internet experience, the use of alternate remote purchasing methods (such as telephone and mail-order shopping), the perceived risks of online shopping, and online purchasing activity. Implications for online commerce and consumer welfare are discussed[5]. Mauricio S. Featherman, Paul A. Pavlou(2003) extends that approach to include measures of negative utility (potential losses) attributable to e-service adoption. Drawing from Perceived Risk Theory, specific risk facets were integrated, and empirically tested within the Technology Acceptance Model resulting in a proposed e-services adoption model[6]. Nadia Oliveroand Perter Lunt's (2001)study shows that the Internet is increasingly being used as a medium to collect information for consumer profiling. However, attempts at data collection face potential difficulties due to consumers' unwillingness to provide personal information. To explore the social and psychological issues underlying consumers' privacy concerns, this paper adopts an interpretative approach. Participants took part in long qualitative interviews through repeated e-mail exchanges. Interview data were combined with insights from the literatures on self-disclosure, sociology, and consumer research in a grounded theory analysis. The analysis indicates that perceived risk and awareness of information collection/extraction are associated with a shift in concerns from issues of trust to issues of control. Risk awareness reduces the level of trust and increases the demand for 
control and rewards complicating the relationship between the retailer and the consumer ${ }^{[7]}$. David Gefen(2000) said trust is a prerequisite of social behavior, especially regarding important decisions. This study examines this intriguing idea in the context of the Ecommerce involved in inquiring about and purchasing books on the Internet. Survey data from 217 potential users support and extend this hypothesis. The data show that both familiarity with an Internet vendor and its processes and trust in the vendor influenced the respondents' intentions to inquire about books, and their intentions to purchase them. Additionally, the data show that while familiarity indeed builds trust, it is primarily people's disposition to trust that affected their trust in the vendor. Implications for research and practice are discussed ${ }^{[8]}$. Tamara Dinev, Massimo Bellotto, Paul Hart, Vincenzo Russo, Ilaria Serra and Christian Colautti(2006) examined cross-cultural differences beliefs related to ecommerce use for Italy and the United States. We argue that for both cultures, the user's decision to make an online purchase is simultaneously influenced by a set of contrary factors. These include decision facilitators such as propensity to trust and institutional trust, and decision inhibitors such as perceived risk and privacy concerns. We argue that substantial cultural differences exist that affect the above factors and the relationships among them. We use Hofstede's cultural theory and Fukuyama's theory of trust and social capital, along with emic factors important for the Italian society, to develop the study's propositions. The hypotheses were empirically tested using LISREL structural equation modeling and multigroup analysis. The results revealed that the Italian society exhibited lower propensity to trust, institutional trust, privacy concerns, and higher perceived risk. The relationships between institutional trust and e-commerce use, privacy concerns and e-commerce use, and perceived risk and institutional trust are all weaker for Italy. The relationship between perceived risk and privacy concerns is stronger for Italy. The paper's major contribution is in validating an important model of e-commerce use across two cultures and showing the moderating effects of culture ${ }^{[9]}$

\section{Analysis of Formation Factors}

E-commerce perceived credit risk is: in e-commerce, online transactions subject perceive the possibility of the other does not comply with the contract (or be deceived). Perceived credit risk arises from objective credit risk in the electronic commerce activity, it is a combination product of credit risk and traders objective subjective feelings in the process of electronic commerce. The formation factors are classified into three parts as: buyers factors, sellers factors and environment factors, which are shown in Table 1.

\subsection{Buyers Factors}

Buyer factors refer to personal characteristics and consumer-related factors, which includes the number of successful transactions, deceived times, the amount of deception and personal prevention capability. A large number of successful transaction can reduce the buyer's perceived credit risk. Be deceived experience is an important factor affecting the traders on the perceived trust risk in e-commerce, which is not only related with the deceived times, also with the deceived amount. Personal defense ability also have obvious influence on the perceived credit risk, the buyer with poor defense ability often amplify the risk. Because even under the same technic and environment conditions, different buyer might look the perceived credit risk as different level, buyers factors therefore is called dynamic factors. 


\subsection{Sellers Factors}

The seller can directly influence the perceived credit risk, includes registration time, credit rating, the commodity price, the transaction amount, payment security and other consumer evaluation on the seller's. Generally speaking, the longer the registration time is and the higher the credit rating is, then the higher perceived credit could be. Consumers evaluation could be classified into tow classes as: favorable and negative, or positive evaluation and negative evaluation, it is worthwhile to note that judge of favorable and negative is with different weight impact on perceived seller credit, the impact of negative evaluation is usually much more stronger than the positive one. Commodity price will also affect the perceived credit risk, buyers would be more cautious when buy valuable commodity through network electronic commerce platform. The reason why commodity price and the total amount of transaction are looked as two different factors is that many buyers feel greater credit risk in the primary trade, they might prefer to buy a small amount products at the first time, after the first successful transaction they make place a large order. Affect of payment security is significant on credit risk perception in electronic commerce activities, cash on delivery is thought as a much more safe way than bank transfer.

\subsection{Trading Environment Factors}

Trading environment factors include e-commerce fraud ratio and fraud crackdown. Low fraud ratio and high-profile fraud crackdown would make the buyers feel safe to place an order through network e-commerce platform. High fraud ratio does not mean it is definitely to be cheated while place an order online, but it represents the incidence rate of deceive. High fraud ratio is like a kind of warning, which makes the potential buyer who never been cheated or never tried e-commerce feel not safe to place an order online. Fraud crackdown is the opposite way to lead the potential buyers' feeling. If internet fraud is crackdown with high cost of breaking the low, every potential would feel much safer.

\section{Analysis of Control Factors}

Control factors could be classified into two parts as technical factors and non-technical factors shown in Table 1.

\subsection{Technical Factors}

Perceived credit risk caused by technical factors mainly depends on the status of network security and the security situation of e-commerce platform. Network security includes the firewall technology, digital signature technology, data encryption technology, security system includes user login and recognition, online payment system security, database security and so on. In the actual environment, good trading habits may be more useful to improve risk prevention than purely technical factors, but quite a few buyers believe that advanced computer and network technology plays a more important role than personal safety consciousness.

\subsection{Non-technical Factors}

Non-technical factors include commitment to return and cash on delivery. Commitment to return is a kind of guarantee that if what the consumer bought was not what he wanted, or not good enough as he supposed, then he could return the product to the seller and get refund. Under normal circumstances, it does not occur very often to return the product and get refund, 
but it is like a kind of insurance to keep the consumers' right when it actually happens. Cash on delivery is an old way to do the payment, however, it is still quite popular in China and India. The social credit system is not well built in China, the potential online buyers always feel not safe to transfer online, so cash on delivery is a good way for them, further more, cash on delivery is another guarantee for them to get the supposed product, because they could check the product before the payment.

Table 1. Formation and Control Factors

\begin{tabular}{|c|c|c|c|}
\hline \multirow{16}{*}{$\begin{array}{l}\text { Electronic } \\
\text { Commerce } \\
\text { Perceived Credit } \\
\text { Risk Formation and } \\
\text { Control Factors }\end{array}$} & \multirow{12}{*}{ Formation Factors } & \multirow{4}{*}{ Buyers Factors } & successful transactions \\
\hline & & & deceived times \\
\hline & & & amount of deception \\
\hline & & & personal prevention capability \\
\hline & & \multirow{6}{*}{ Sellers Factors } & registration time \\
\hline & & & credit rating \\
\hline & & & commodity price \\
\hline & & & transaction amount \\
\hline & & & payment security \\
\hline & & & consumer evaluation \\
\hline & & \multirow{2}{*}{ Environment Factors } & e-commerce fraud ratio \\
\hline & & & fraud crackdown \\
\hline & \multirow{4}{*}{ Control Factors } & \multirow{2}{*}{ Technical Factors } & network security \\
\hline & & & platform security \\
\hline & & \multirow{2}{*}{ Non-Technical Factors } & commitment to return \\
\hline & & & cash on delivery \\
\hline
\end{tabular}

\section{Model of e-commerce Perceived Credit Risk}

A system dynamic model is built to analyze the formation and control mechanism of ecommerce perceived credit risk, because e-commerce perceived credit risk is shown as a complex system, a system dynamic model is suitable to deal with it.

\subsection{Determination of the Boundaries of the System}

It is believed in system theory that the integrity of the system is a special boundary reaction which appears when the system contact with the environment. This is because that there are some special coherence contacts between the system internal elements. When each element in the interaction with the external environment, the contact performance as the overall effect of the system which affect the environment. When material, energy and information get into the system from the outside world or get out of the system to outside world, function mutations appear between the system boundaries. The boundary of the system, play the role of protecting the stability of the system under certain conditions. The system boundary is determined as perceived risk factors of formation control factors and the interaction of various factors based on buyers perspective in e-commerce activities. 


\subsection{Causal Relationship Diagram}

Causal relationship diagram of e-commerce perceived credit risk is shown in Figure 1. Ecommerce perceived credit risk could be increased by buyers factors, sellers factors and environment factors. High perceived credit risk would decrease buyers purchase intention, however, the demand for e-commerce is keeping increasing because the popularity of the network, so low buyers purchase intention could increase risk control factors.

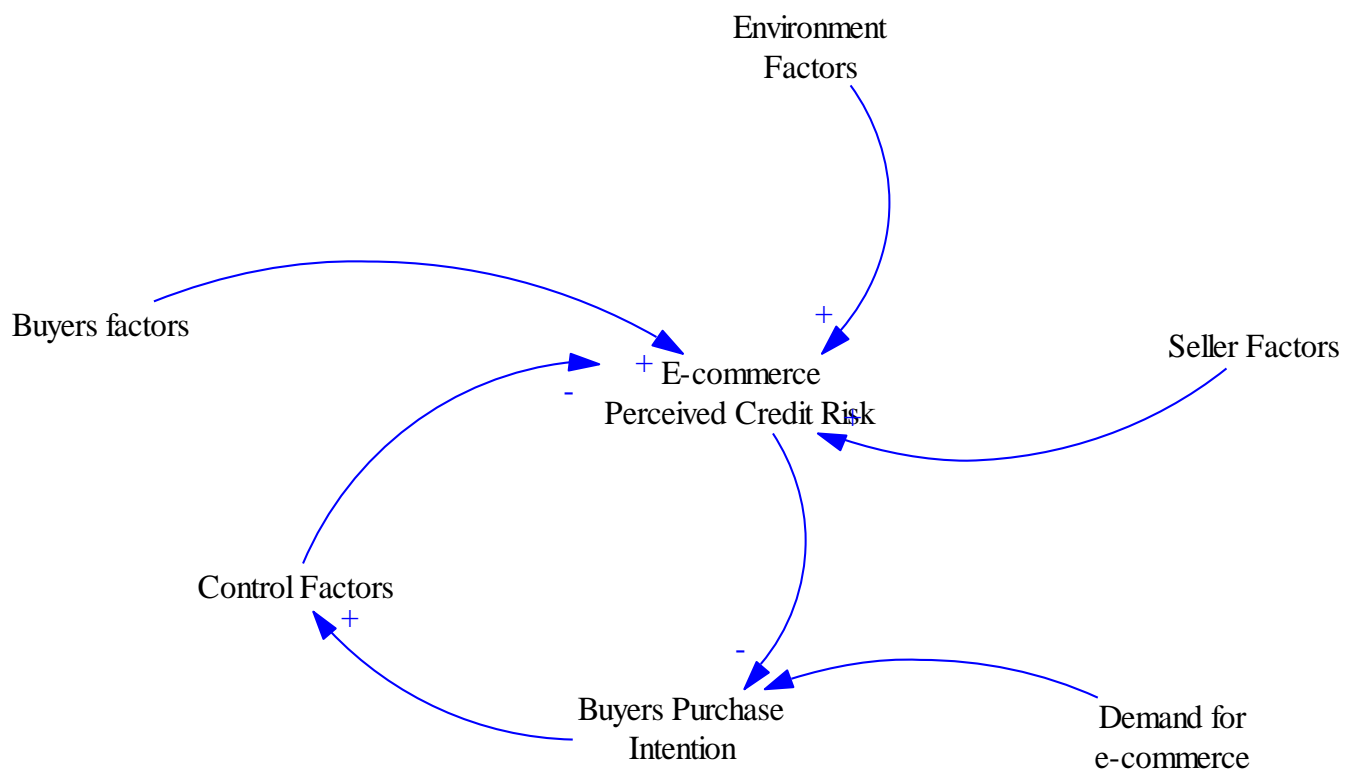

Figure 1. Causal Relationship Diagram

\subsection{System Dynamic Model}

System Dynamics is a systematic approach combined of qualitative and quantitative to deal with complex issues, it combines the feedback control theory, information theory, systems theory, decision theory, computer simulation, and system analysis method ${ }^{[10]}$. The ecommerce perceived credit risk system is a dynamic complex system with both integrity and dynamic characteristics, and also the behavior of complex systems of non-linear, higher order and multi-loop ${ }^{[11]}$. Only the basic structure is shown in figure 1 , it is impossible to tell the distinction of the different nature of variable. It is necessary to use the flow graph to create a complete system dynamics model. By refining the causality of e-commerce perceived credit risk and introducing the characterization indicators of the main variables, e-commerce perceived credit risk model based on system dynamics is built shown as Figure 2. Ecommerce perceived credit risk is represented as state variable, buyers factors, sellers factors, environment factors and control factors are represented as rate variable, the others are auxiliary variables and exogenous variables. 


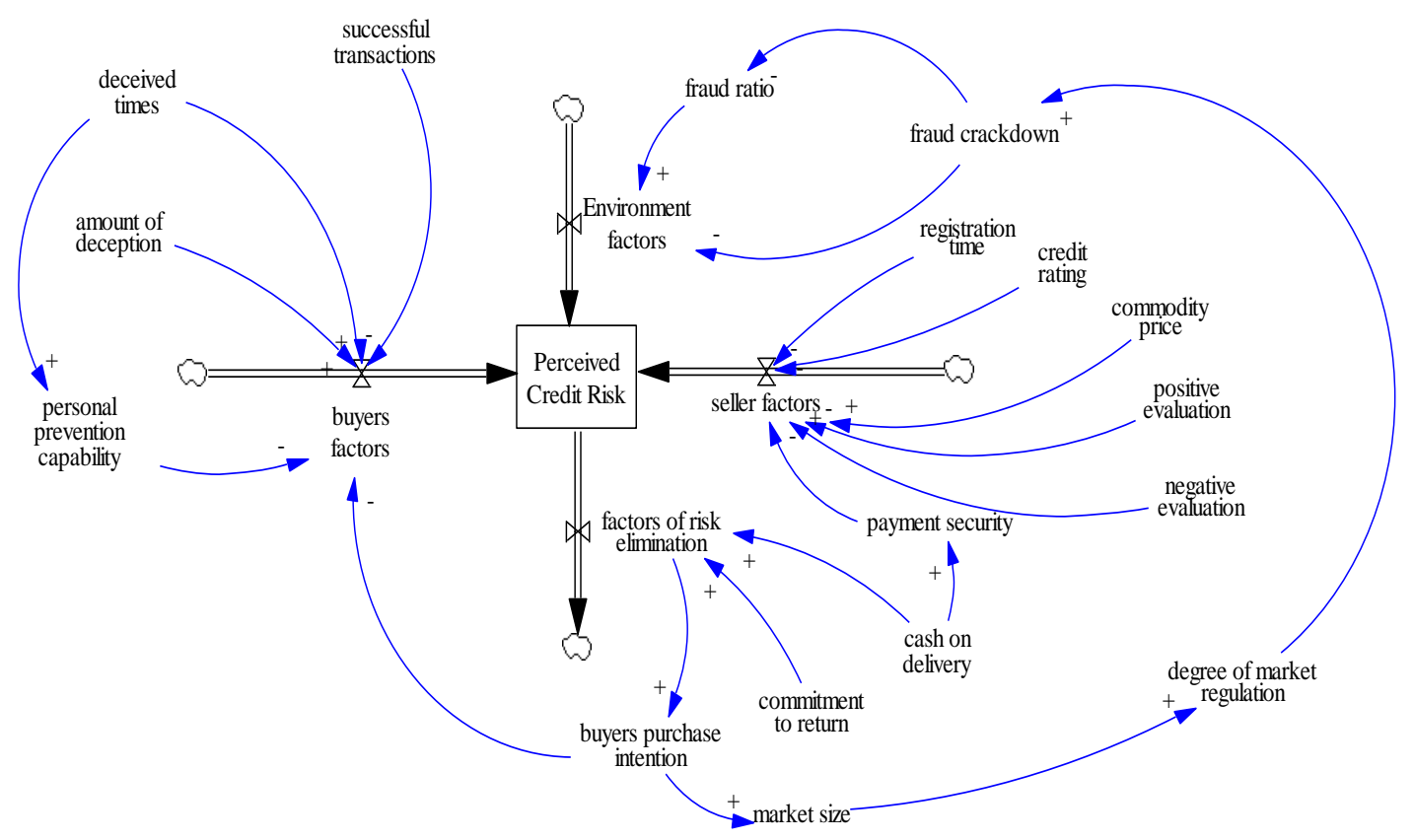

Figure 2. Flow Graph of System Dynamic Model

As shown in Figure 2, perceived credit risk is directly influenced by four factors which are buyers factors, seller factors, environment factors and factors of risk elimination. Factors os risk elimination is the way to decrease perceived credit risk, the other three are the factors to increase perceived credit risk. Successful transactions would decrease buyers factors, it means the more times you purchase successfully online, the less perceived credit risk you would feel in your mind while making an order online. Deceived times and amounts would increase buyers factors, if a buyer has deceived plenty of times he might give up purchase online at all. At the mean while, deceived times could also increase personal prevention capability which is a factor influence the buyers factor in an opposite way. Seller factors could be influenced by quite a few elements, the longer the registration time is, the lower perceived credit risk caused by seller factors could be felt, it is exactly like we purchase in the real world, we always believe a store with long history could provide better products. Credit rating is a sign to show how many transactions a online store has made and how good it served the clients, it has positive influence to the online store. Commodity price could also influence the perceived credit risk, when a buyer chooses a product with high price he could be more vigilant, otherwise, if the product is cheap he might not be worried about bing cheated. The evaluation by the previous buyer is a good way to judge credit risk, evaluation could be classified into positive ones and negative ones which show the satisfaction and dissatisfaction of previous buyers. Positive evaluation could decrease the perceived credit risk, the negative evaluation could increase the perceived credit risk.

\section{Conclusion and Suggestions}

\subsection{Establish e-commerce Credit Information Database}

The appearance of faithless conditions is mainly because of information not shared, which leads to asymmetric information and the merchants' adverse selection. The trading characteristics of e-commerce "not met" greatly aggravate the probability of the 
adverse selection occurrence. Aiming atthe situation, building a good information sharing mechanism by the construction of social credit system is necessary. Through the establishment of the province electronic commerce social credit guarantee system, make electronic commerce faith of individuals and enterprises become the sharing information. Establishing roundly faithless "blacklist" system and sharing faithful information can transform the trading or exchanges into infinite and continuous game, thus increasing the opportunity cost of electronic commerce in bad faith. In building the social credit e-commerce security system, we should standardize the procedures of information collection, share credit resources and improve the information management.

\subsection{Arouse the Market Enthusiasm, and Guide the Development of the Third Party Credit rating Agencies}

Positively, develop the credit intermediary organ and establish specialized and market-based credit management services. Enterprises apply voluntarily, which will greatly improve the executive efficiency of e-commerce and promote its long-term development. Such services provided by the credit services include credit investigation, credit rating, credit insurance and business account recovery etc.. It constitutes the social credit management support system, and becomes the important part of effective operation in the social credit guarantee system.

\subsection{The Faithless Punishment Mechanism}

Pursuing maximization of gain is the faithless economic root, and curbing the faithless people must build the effective system and proceed to the external constraints. Faithless enterprise or individual will be subject to administrative or judicial punishment and disclosed in the relevant economic and social area, which have a negative impact on the survival and development.We should establish faithless disciplinary mechanism as soon as possible, and make serious faithless behavior punished and let the faithless cost become the price that cannot afford, so that credit consciousness of enterprises and civil will continue to improve, then constantly improve credit system.

\subsection{Build Traceability Mechanism of Food, Pharmaceutical and Other Special Product}

The government should perfect the relevant laws and regulations on the one hand, and insert the mandatory traceable system in the online circulation link of food and pharmaceuticals; On the other hand, as for the investment and various fees in the process of establishing traceable system,the government should increase the support to the upstream part whose ability of burdening and bearing is weak through industrial preferential policy,encouraging food, medicine, electronic commercial platform to participate in the safety traceability system construction.

\subsection{Build Integrity Electric Goods Brand of China}

The government leads to form China credit electricity union, integrate the low profile, the lack of customer credit electric business platform, the government and the third party credit rating agencies to guarantee "alliance" electric business platform in the credit, dispel consumers' concerns for the credit risk, and make faithful brand effect. 
E-commerce perceived credit risk is the key factor in the decision-making of consumers, thus the risk formation and control analysis is the route one must take measures to promote the development of e-commerce. It is very important to analyze and control e-commerce perceived credit risk correctly. The characteristics of ecommerce system are considered on the basis of domestic and foreign research, the system dynamic model is built with full consideration of buyers' factors, sellers' factors, environment factors and control factors. The analysis in this paper is the basis for further research such as: risk evaluation, prevention and control strategies development.

\subsection{Government Support and Hatching Quality Enterprises}

Many multinational companies require e-commerce outsourcing service providers own corresponding international certificate (CMM, CMMI), and to obtain these certifications require a significant amount of human and financial resources, which for some promising young companies is undoubtedly a heavy burden. If the Government can establish a special support funds, incentives for certified enterprises, it can effectively support enterprise adoption of seriously and earnestly strengthen their own management capacity, adding to its international competitiveness. Bigger and stronger enterprises expand employment, and increase profits and taxes to support local financial and spur economic development.

\section{Acknowledgements}

This paper is sponsored by Heilongjiang Province philosophy social science research projects fund No. 13D031, Heilongjiang Provincial Department of Education Social Sciences Project No. 12532069, Financial Assistance under Heilongjiang Postdoctoral Fund No. LBHZ12146, Phd Startup Project of Harbin University of Commerce, and National Natural Science Fund No. 61003036.

\section{References}

[1] Y. Liebermann and S. Stashevsky, "Perceived risks as barriers to Internet and e-commerce usage", Qualitative Market Research: An International Journal, vol. 5, (2002), pp.291 - 300.

[2] D. J. Kim, D. L. Ferrin and H. R. Rao, "A trust-based consumer decision-making model in electronic commerce: The role of trust, perceived risk, and their antecedents", Decision Support Systems, vol. 44, (2008),pp. 544-564.

[3] C. L. Nicolas and F. J. M. Castillo, "Customer Knowledge Management and E-commerce: The role of customer perceived risk", International Journal of Information Management, vol. 28, no. 2, (2008),pp. 102113.

[4] C. Park and J. K. Jun, "A cross-cultural comparison of Internet buying behavior: Effects of Internet usage, perceived risks, and innovativeness", International Marketing Review, vol. 20, no. 5, pp.534 - 553.

[5] A. D. Miyazaki and A. Fernandez, "Consumer Perceptions of Privacy and Security Risks for Online Shopping”, Journal of Consumer Affairs, vol. 35, no. 1, (2001), pp. 27-44.

[6] M. S. Featherman and P. A. Pavlou, "Predicting e-services adoption: a perceived risk facets perspective", International Journal of Human-Computer Studies, vol. 59, no. 4, (2003), pp. 451-474.

[7] N. Olivero and P. Lunt, "Privacy versus willingness to disclose in e-commerce exchanges: The effect of risk awareness on the relative role of trust and control. Journal of Economic Psychology", vol. 25, no. 2, (2004), pp. 243-262.

[8] D. Gefen, "E-commerce: the role of familiarity and trust", Omega, vol. 28, no. 6, (2000), pp. 725-737.

[9] T. Dinev, M. Bellotto, P. Hart, V. Russo, I. Serra and C. Colautti, "Privacy calculus model in e-commerce - a study of Italy and the United States", Regular Article European Journal of Information Systems, (2006), pp. 389-402.

[10] M. K. Gilley, "Making More by Doing Less: an Analysis of Outsourcing and Its Effects on Firm Performance", Journal of Management,(2000). 
[11] M. Beasley, M. Bradford and D. Pagach, "Outsourcing at Your Own Fisk”, Strategic Finance, (2004).

\section{Author}

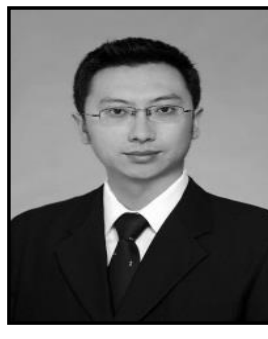

En-lin Li, Associate professor.Main research fields : Information Service; E-commerce and E-government. This paper is sponsored by Heilongjiang Province philosophy social science research projects fund No. 13D031, Heilongjiang Provincial Department of Education Social Sciences Project No. 12532069, Financial Assistance under Heilongjiang Postdoctoral Fund No. LBH-Z12146, Phd Startup Project of Harbin University of Commerce, and National Natural Science Fund No. 61003036. 